\title{
THE ATMOS SOLAR ATLAS
}

\author{
CROFTON B. FARMER \\ Jet Propulsion Laboratory, California Institute of Technology, \\ Pasadena, CA 91109, U.S.A.
}

\begin{abstract}
The ATMOS solar atlas covers the infrared spectrum of the Sun observed from space, over the frequency range from 650 to $4800 \mathrm{~cm}^{-1}$ at a resolution of $0.01 \mathrm{~cm}^{-1}$. The spectrum reveals a large number of molecular and atomic features that are not visible in spectra taken from the ground. The acquisition of the spectra, details of their presentation in the atlas, and some highlights of the solar features are described.
\end{abstract}

Key words: artificial satellites, space probes - infrared: stars - instrumentation: interferometers - Sun: atmosphere

\section{Introduction}

The ATMOS solar infrared atlas (Farmer and Norton, 1989) was compiled from spectra obtained during the Spacelab-3 mission in April/May 1985. These observations, which were part of a continuing program of simultaneous measurements of the vertical profiles of the minor and trace gases in the earth's upper atmosphere, were made by recording the solar-telluric absorption spectrum at a spectral resolution of $0.01 \mathrm{~cm}^{-1}$ throughout the near and mid-infrared wavelength region, during sunrise and sunset occultation periods as seen from the Space Shuttle.

In order to be able to clearly identify the solar and any residual instrumental or spacecraft-associated spectral features, and remove them from the spectrum of the earth's atmosphere, the observations were designed to include - at the beginnings of the sunset occultations, and at the ends of the sunrises - a period during which spectra would be recorded with the tangent point of the Sun-spacecraft line well above the sensible atmosphere. After careful examination of the spectra, it was determined that no absorption features of atmospheric origin could be detected above about $150 \mathrm{~km}$ altitude, so that an altitude some two scale-heights above this (i.e., $165 \mathrm{~km}$ ) was defined as the top of the atmosphere for this purpose. Spectra obtained at tangent- point altitudes above $165 \mathrm{~km}$ were assumed to contain only features of solar or instrumental origin. Thus the program of detailed measurements of the infrared- active constituents of the earth's atmosphere provided the opportunity to record a large number of high resolution spectra of the Sun free from interfering telluric lines, covering the continuous wavelength range from 2 to $16 \mu \mathrm{m}$. These spectra, co-added to give a single average spectrum having a signal-to-noise ratio on the order of 2000 to 1 , formed the basis of the solar reference spectra reproduced as Vol. 1 of the ATMOS atlas. A brief description of those aspects of the design of the instrument and the observations pertinent to the characteristics of the solar spectrum is given below.

\section{The $A T M O S$ Instrument}

The requirement to make measurements of the vertical distributions of trace species throughout the stratosphere and mesosphere, with a vertical resolution of a few $\mathrm{km}$, dictated the need to cover the 2-16 $\mu \mathrm{m}$ range at a spectral resolution of $10^{-2} \mathrm{~cm}^{-1}$ 
in a scan time of about 1 second. (The rate of change of the tangent height of the Sun-spacecraft line of sight for a typical shuttle orbit is about $2 \mathrm{~km} \mathrm{~s}^{-1}$.) The resulting instrument design specified a double-passed Michelson interferometer in which both retroreflectors ("cat's eyes") move in a parallel, reciprocal motion, symmetrical about the zero path difference (zpd) point. There are thus four singlesided interferograms in a total scan cycle of $4.2 \mathrm{~s}$; data from the transformed singlesided scans sharing a common zpd were averaged, providing occultation sequences in which the effective altitudes of the individual spectra were spaced by about $4 \mathrm{~km}$. While the minimum detectable equivalent width (MDEW) of features in the atmospheric spectra is not better than about $10^{-4} \mathrm{~cm}^{-1}$ (corresponding to a $S / N$ ratio of 100:1), the relatively rapid scan time necessitated by the requirements of the atmospheric spectra works in favor of the solar spectra in the sense that many scans can be acquired in the time available at the ends of the occultations, resulting in an order of magnitude improvement in the MDEW of the final averaged solar spectra.

To optimize the measurements in terms of instrument performance and data rate under orbital conditions, the overall frequency range was divided into four narrower bands by means of optical filters. The bandpasses of the filters were chosen to be compatible with the alias limits of sampling at every second or every third fringe of the reference $\mathrm{He}: \mathrm{Ne}$ laser, i.e., 600-1200, 1100-2000, 1580-3400 and 3100$4700 \mathrm{~cm}^{-1}$. The first three filters were used with a sampling interval of two laser fringes; for the fourth filter the interferogram was sampled at every third fringe. The instrument, which was built by Honeywell Electro-Optics Center, consists of four main parts: the suntracker, the foreoptics and frame camera, the interferometer and scan control system, and the electronics subunits, which include the closed-cycle detector cooler. Figure 1 is a schematic diagram of the optical system.

An important aspect of the instrument design, particularly in terms of its effect on the solar spectra, was the use of a thin aluminum cover which attached to the baseplate, enclosing all but the suntracker and camera. The pressure within the cover was maintained at ambient by means of a vent fitted with a dessicant filter designed to provide a clean, dry environment under all conditions (and, in particular, to protect the $\mathrm{KBr}$ beamsplitter and compensator during reentry into the atmosphere). This filter prevented complete evacuation of the air inside the cover, even after several days in orbit, so that residual lines of $\mathrm{H}_{2} \mathrm{O}$ and $\mathrm{CO}_{2}$ appear in all of the spectra. These lines fortuitously provide a valuable frequency reference for the rest frame against which the relative Doppler shifts of the solar lines could be verified. Although the numbers of molecules of water vapor and carbon dioxide were sufficient to cause the superimposed instrumental lines (about $2.5 \times 10^{17}$ and $2.7 \times 10^{15} \mathrm{~cm}^{-2}$, respectively), the internal instrument pressure was too low to require an air-to- vacuum frequency correction to the data. The frequency stability of the instrument during the observations is estimated to be equivalent to an overall uncertainty of $3 \times 10^{-4} \mathrm{~cm}^{-1}$ in the measurement of the frequency of a single line in a single spectrum.

In addition to the lines produced by the residual gas, several other absorptionlike features of instrumental origin are superimposed on the solar and atmospheric spectra. These "artifacts", which are perhaps due to contamination of the surfaces 


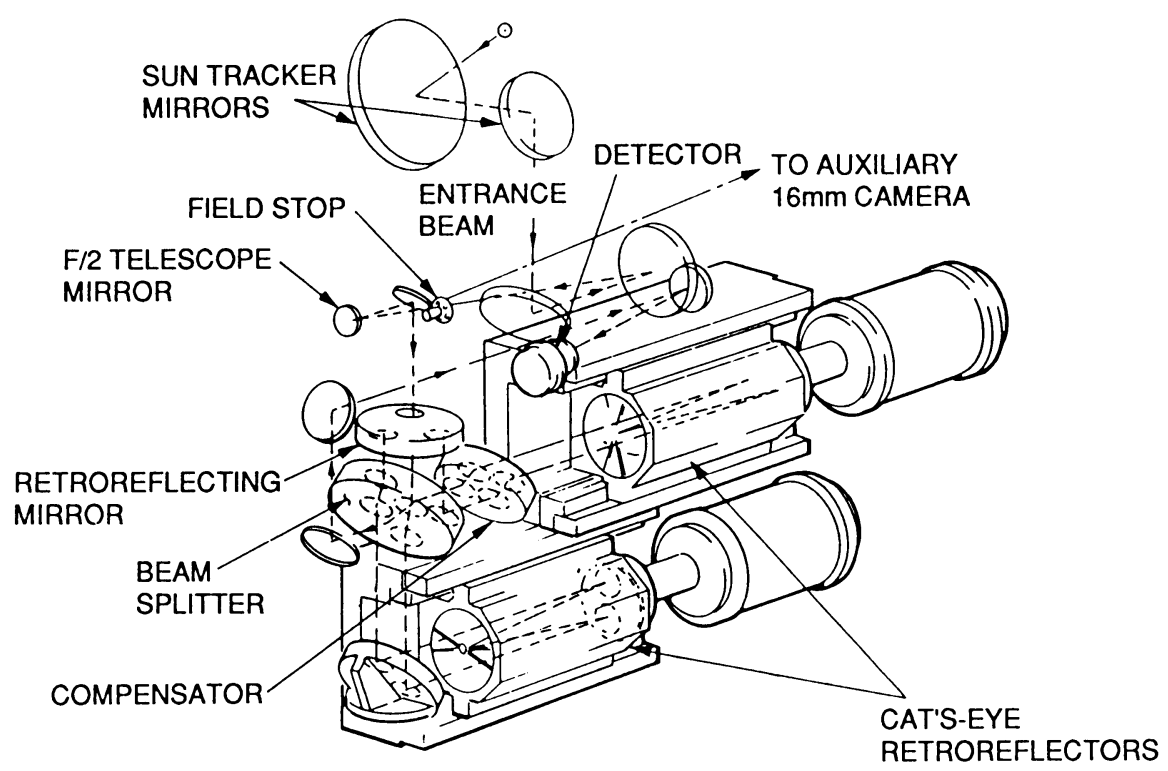

Fig. 1. Layout of the key optical components of the ATMOS interferometer.

of the optical components, can be distinguished from the residual gas lines by their width; whereas the latter appear at the instrument resolution $\left(0.01 \mathrm{~cm}^{-1}\right)$, the artifacts are typically about $1 \mathrm{~cm}^{-1}$ wide.

The instrument views the Sun through a selectable field stop of 1,2 , or $4 \mathrm{mrad}$. which can be positioned with respect to the center of the disc in steps of $1 \mathrm{mrad}$. Thus coarse limb scans can be performed, or scans accumulated at chosen radial distances, although these options were not exercised during the first flight of the instrument. The suntracker is able to acquire and track the Sun over the entire hemisphere above the wing plane of the Shuttle; the tracking accuracy is $0.4 \mathrm{mrad}$, with a stability of $0.06 \mathrm{mrad}$.

\section{Data Reduction}

Details of the procedure used for transformation of the recorded interferograms to spectra are given in the Data Reduction section of Volume I of the Atlas. The point spacing of the transformed data is $0.00753 \mathrm{~cm}^{-1}$, for filters 1,2 and 3 , and $0.00502 \mathrm{~cm}^{-1}$ for filter 4 . The spectra were apodized (using function number 2 of Beer and Norton, 1976) and interpolated with four additional points between the primary points; the point spacings for the final spectra are thus $0.001506 \mathrm{~cm}^{-1}$ and $0.00100 \mathrm{~cm}^{-1}$.

The variation of the Sun-spacecraft radial velocity during the acquisition of the solar scans was small, as these were taken at the highest tangent altitudes; 
consequently, all of the solar spectra could be averaged together on a point-bypoint basis to produce a solar reference spectrum for each occultation. The validity of this procedure was tested by cross-correlation of the individual spectra with the average. The high correlation coefficients found (see the descriptive text of the atlas for details of these results) provide a direct indication of the quality of the individual spectra making up the averages. A similar point-by-point averaging procedure was then used to generate "zonal average" spectra, or grand averages of all of the spectra obtained with each optical filter and at the same radial velocity (i.e., a sunrise and a sunset average for each filter). Calculation of the cross-correlation coefficients between the occultation averages and the corresponding grand average again gave very high values ( 0.999944 in the worst case), because changes in the radial velocity resulting from changes in the orbital geometry over the two days during which data were acquired were too small to produce a significant spread in the frequency shift. Finally, in order to minimize any ambiguity in the identification of lines, the sunrise and sunset averages are displayed in the atlas with their frequencies corrected for the component of the relative velocity along the Sun-spacecraft line. In this way, lines whose positions appear shifted between the sunrise and sunset spectra are of instrumental origin and are easily distinguished.

\section{Description of the ATMOS Solar Spectrum}

In all, some 16,000 solar features appear in the ATMOS atlas, the majority of which are lines of the vibration-rotation bands of the diatomic molecular constituents of the photosphere: $\mathrm{CO}, \mathrm{CH}, \mathrm{OH}$ and $\mathrm{NH}$. Of these, the $v=1$ and $v=2$ bands of $\mathrm{C}^{12} \mathrm{O}^{16}$ and of its isotopic variants dominate the spectrum as a whole, contributing more than a half of all of the lines of solar origin. In addition to the molecular lines, about 1700 atomic lines are present, due mainly to transitions in neutral $\mathrm{Fe}, \mathrm{Si}$, $\mathrm{Mg}, \mathrm{C}, \mathrm{Ca}$, and $\mathrm{Al}$. The atomic features represent about $11 \%$ of the total number of lines in this region of the infrared solar spectrum; almost 4000 lines (or $24 \%$ of the total) remain unidentified. The preparation of a key, listing the frequencies and identifications of the lines in the ATMOS spectrum, has been undertaken by M. Geller of JPL (Geller, 1992). This key has been submitted for publication as Volume III of the ATMOS atlas series. Table 1, taken from the key, summarizes the catalogued lines and their identifications. Note that $\Delta v=1$ entries for $\mathrm{CO}$ include lines arising in $\mathrm{C}^{12} \mathrm{O}^{16}, \mathrm{C}^{13} \mathrm{O}^{16}, \mathrm{C}^{12} \mathrm{O}^{18}$; those for $\Delta v=2$ are for $\mathrm{C}^{12} \mathrm{O}^{16}$ only.

\subsection{MOLECULAR TRANSITIONS}

Figure 2 shows an example of a portion of the spectrum in the region of the bandheads of the $v=1$ bands of $\mathrm{CO}$. The $\mathrm{CO}$ bands present a striking appearance in the solar spectrum seen without any interference from atmospheric absorptions, starting at $2328 \mathrm{~cm}^{-1}$ and progressing to lower frequencies, remaining visible to below $1400 \mathrm{~cm}^{-1}$. With the instrumental $\mathrm{CO}_{2}$ and $\mathrm{H}_{2} \mathrm{O}$ lines clearly distinguishable, the high $S / N$ ratio of the ATMOS spectra allows the sequences to be followed as far as $v^{\prime \prime}=20$ and $J^{\prime \prime}$ as high as 135 . The increased range of rotational quantum values accessible in the spectrum has provided the basis for the revision of the 
1.00
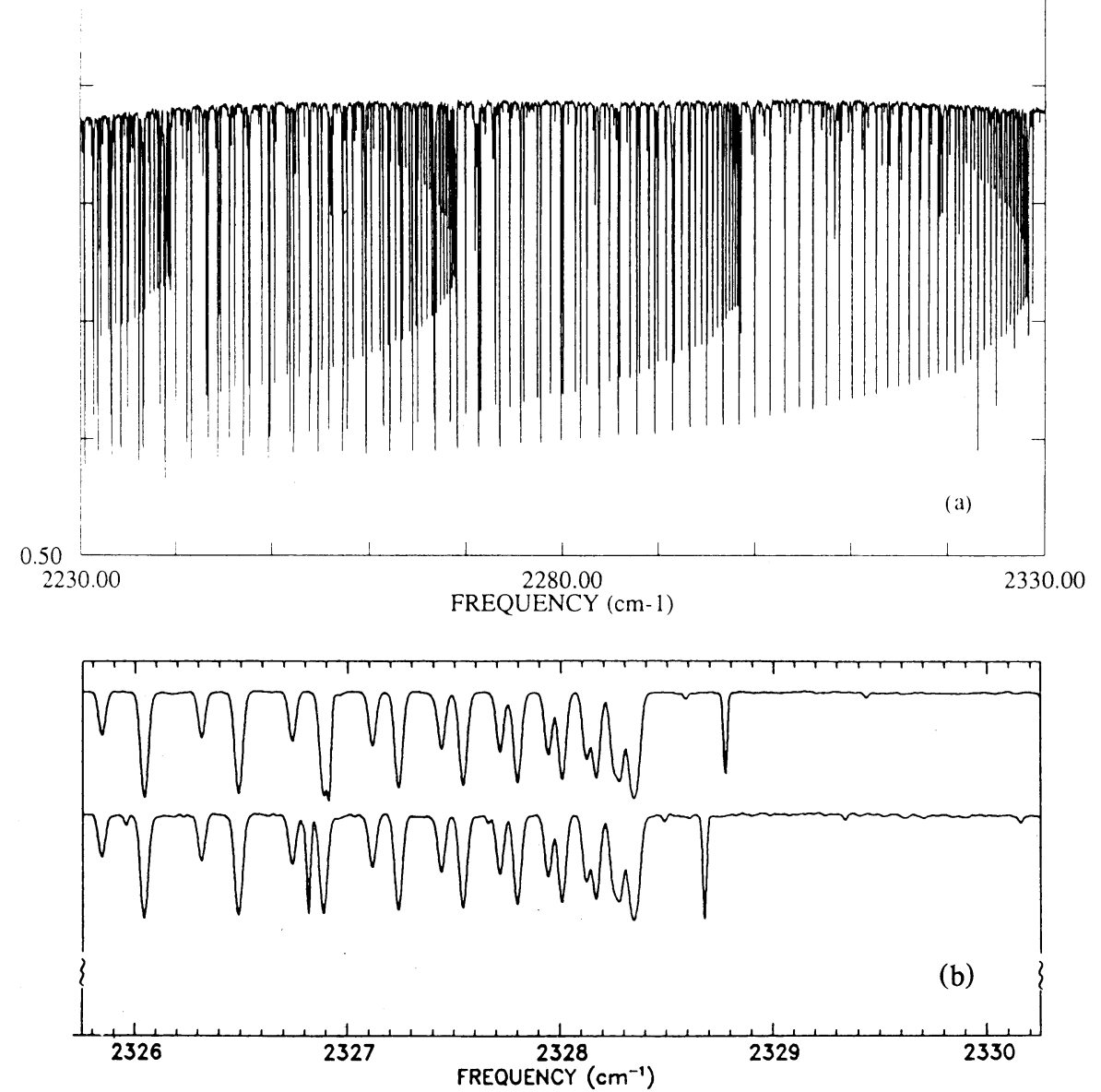

Fig. 2. (a) A low dispersion plot of a portion of the $\Delta v=1$ sequence of $\mathrm{CO}$ bands, showing the first four bandheads; (b) A part of the 1-0 band reproduced at the dispersion of the atlas, illustrating the relative frequency shifts between the instrumental $\mathrm{CO}_{2}$ lines in the sunset (above) and sunrise spectra. 
TABLE I

ATMOS Solar Atlas

Summary of Catalogued Features and their Identifications (Geller, 1992)

\begin{tabular}{ccc}
\hline Identification & $\begin{array}{c}\text { Number of } \\
\text { lines }\end{array}$ & $\begin{array}{c}\text { Percent of } \\
\text { total number }\end{array}$ \\
\hline CO $(v=1)$ & 6478 & 40.1 \\
$\mathrm{CO}(v=2)$ & 2394 & 15.1 \\
$\mathrm{OH}$ & 743 & 4.7 \\
$\mathrm{CH}$ & 581 & 3.7 \\
$\mathrm{NH}$ & 181 & 1.1 \\
Atomic & 1709 & 10.8 \\
Unidentified & 3761 & 23.7 \\
\hline
\end{tabular}

ground-state molecular constants for $\mathrm{CO}$, by the use of the observed frequencies of the high- $J$ lines seen in the spectrum together with available accurate laboratory measurements (Farrenq et al., 1990). All of the $\mathrm{C}^{12}$ isotopes $\left(\mathrm{O}^{16}, \mathrm{O}^{17}\right.$, and $\left.\mathrm{O}^{18}\right)$, as well as $\mathrm{C}^{13} \mathrm{O}^{16}$, are observed in the $v=1$ transitions. For the $v=2$ bands, which cover the range from 4360 to $3330 \mathrm{~cm}^{-1} v^{\prime \prime}$ as high as 16 (for $\mathrm{C}^{12} \mathrm{O}^{16}$ ) can be seen.

For $\mathrm{OH}$, the ATMOS spectra reveal many more of the pure rotational lines (0-0 through 4-4 bands) in the 620 to $1100 \mathrm{~cm}^{-1}$ region than have been visible in ground- based solar spectra (Fig. 3). Some reservations regarding the quantitative fidelity in this region of the spectrum, perhaps resulting from uncorrected nonlinearity in the detector, suggest that caution should be exercised in interpreting the intensities of the $\mathrm{OH}$ lines in terms of the solar oxygen abundance. This matter is being investigated at the present time. In addition to the 370 pure rotation lines, a similar number of vibration-rotation lines of $\mathrm{OH}$ have been identified between 1986 and $3508 \mathrm{~cm}^{-1}$ (Geller, 1992). As in the case of $\mathrm{CO}$, the extended range of frequencies for $\mathrm{OH}$ transitions derived from the ATMOS spectrum is expected to be used to provide improved molecular constants for $\mathrm{OH}$ (Grevesse and Sauval, 1990).

Another diatomic molecule for which the fundamental vibration-rotation lines are excited to much higher rotational levels in the solar spectrum than is possible under laboratory conditions is $\mathrm{CH}$. We have been able to measure a large number of new lines of the 1-0 through 4-3 bands; altogether some 558 lines have been used to derive new molecular constants for the $X^{2} \Pi$ ground state of $\mathrm{CH}$ (Mèlen et al., 1989). From a selected set of these, Grevesse et al. (1991) have derived a solar carbon abundance $(8.60 \pm 0.05)$ which reduces considerably the overall uncertainty associated with values based on measurements in other spectroscopic regions. A portion of the spectrum containing several groups of lines of the fundamental $\mathrm{CH}$ stretching vibration, a region almost totally obscured from ground-based observations by the strong $\nu_{3}$ band of $\mathrm{CH}_{4}$, is shown in Figure 4. In the identification key to the ATMOS solar spectrum, Geller (1992) lists a total of 581 vibration-rotation lines of $\mathrm{CH}$. 


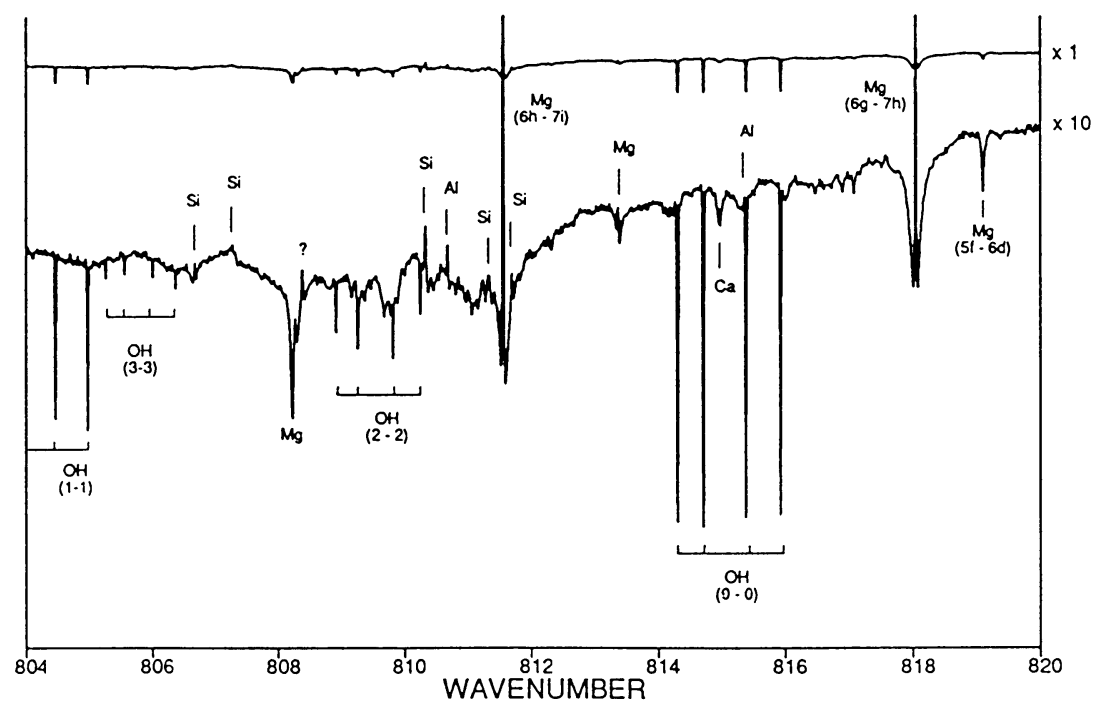

Fig. 3. Pure rotational lines of $\mathrm{OH}$. The intensity scale of the lower trace has been expanded by a factor of 10 .

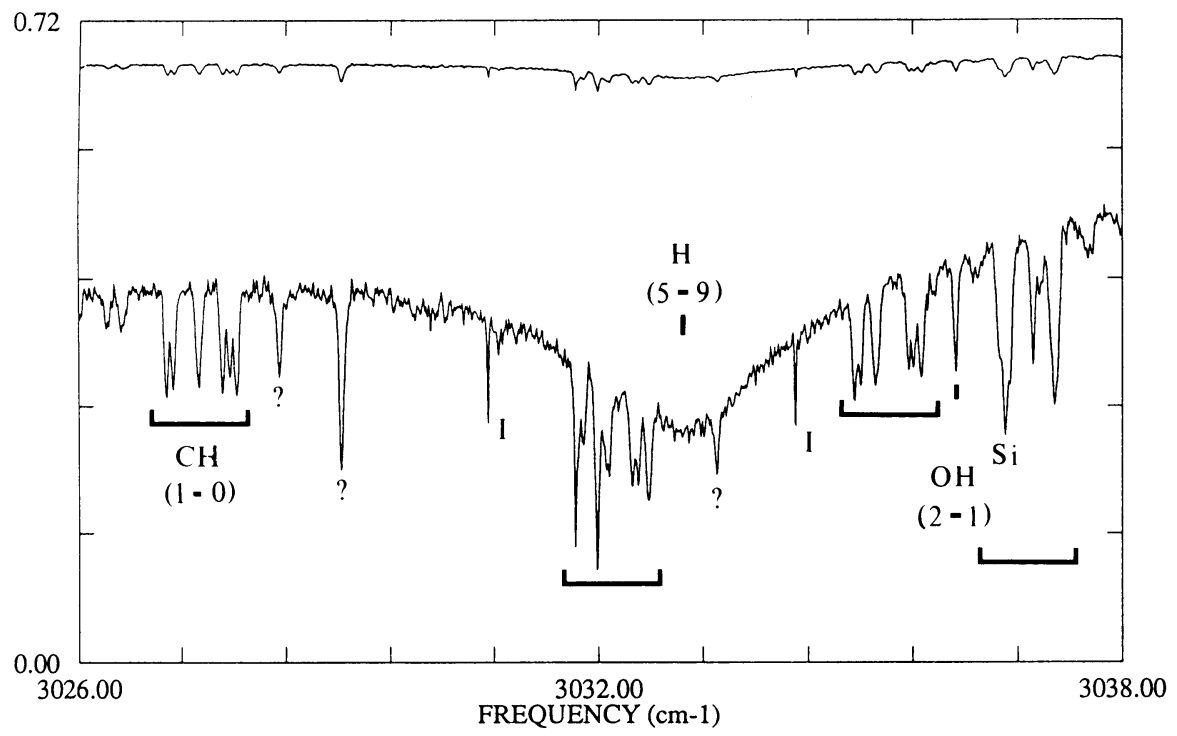

Fig. 4. $\mathrm{CH}$ manifolds in the $3 \mu \mathrm{m}$ region. Lines due to residual gas in the instrument are marked with an I. 


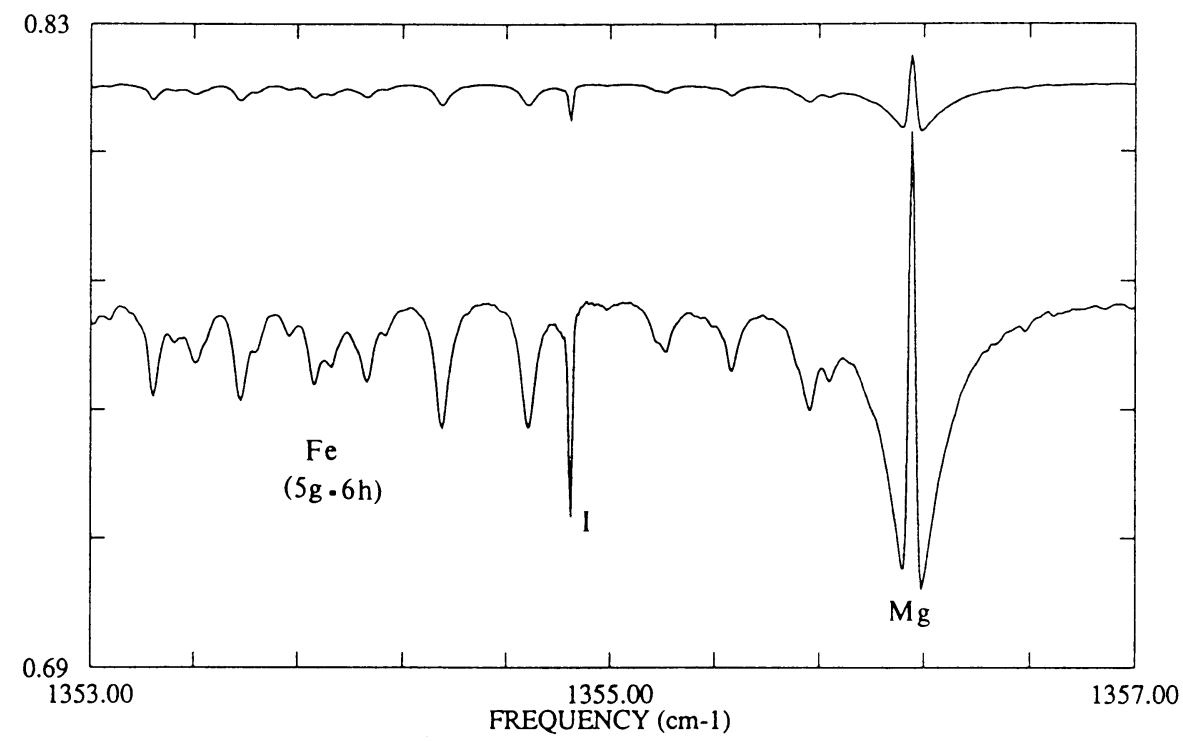

Fig. 5. A cluster of Fe lines in the vicinity of the $\mathrm{Mg}$ I $5 g-6 h$ emission feature.

Both vibration-rotation and pure rotational lines of $\mathrm{NH}$ have been observed for the first time in the ATMOS solar spectrum, as reported by Grevesse et al. (1990) and Geller et al. (1991), respectively. In total, 181 lines of NH (of which 36 are pure rotation lines) are identified in the key to the atlas.

\subsection{Atomic Transitions}

In addition to the molecular lines summarized above, more than 5000 other features, a large fraction of which remain unidentified, appear in the spectrum. The ability to examine the entire frequency range from 600 to $4700 \mathrm{~cm}^{-1}$ without the usual obscuration by broad absorptions in the earth's atmosphere, reveals that many of these features are grouped together in close proximity to the atomic hydrogen transitions. For example, groups of lines occur between 810 and $820 \mathrm{~cm}^{-1}$, 1340 and $1360 \mathrm{~cm}^{-1}$ (shown in Fig. 5), and 2490 and $2530 \mathrm{~cm}^{-1}$, which correspond to the hydrogen $6-7,5-6$, and 4-5 transitions at 808,1340 , and $2468 \mathrm{~cm}^{-1}$, respectively. These include the prominent magnesium emission features observed in earlier ground-based spectra (Brault and Noyes, 1983) and suggested that many of the new lines could also be identified as atomic Rydberg transitions in the light elements. This was confirmed by Jefferies (1991) and Chang et al. (1991), who (along with the authors of several papers in these proceedings) have discussed both the relationship between the frequencies of these transitions and their hydrogenic analogues, and the formation of lines with characteristic emission cores.

In the initial attempts to identify the atomic features, Geller (1992) found that 


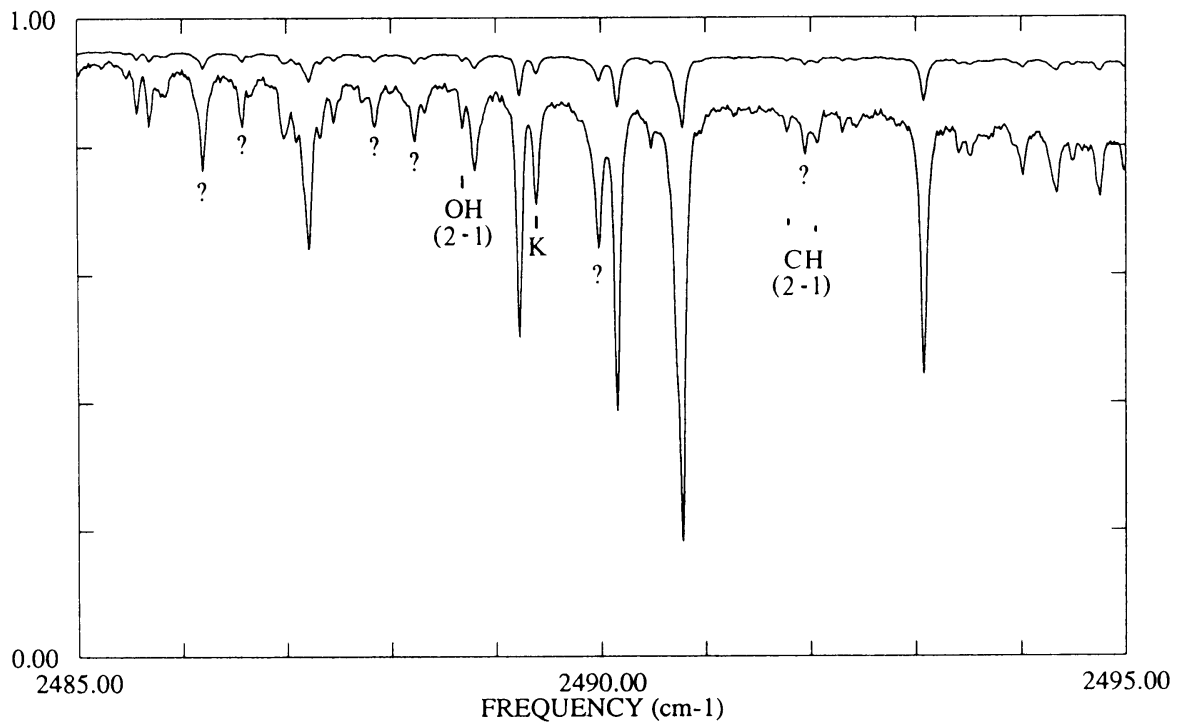

Fig. 6. The strong lines in this interval are Si transitions. Many of the smaller features are not yet identified.

the published compilations of data for the energy levels of the most probable elemental species were insufficient and that further calculations of atomic levels and transitions would have to be performed. As a result of these calculations, most notably for $\mathrm{Fe}$ and $\mathrm{Si}$, by Geller and several collaborators, it has been possible to make unambiguous assignments for more than 1700 atomic transitions in the spectrum. (Details of the new energy levels and of work contributing to these new assignments by Chang, Grevesse, Jefferies, Johansson, Sauval and others can be found in Geller, 1992.)

Other examples of the spectra, in regions largely obscured from the ground, and in which several new atomic features occur, are shown in Figures 6 and 7 . Figure 6 shows a group of strong $\mathrm{Si}$ lines together with a number of other unidentified features. Figure 7 shows a region dominated by $4 f-5 g$ transitions of $\mathrm{Fe}$, with several smaller lines of the higher vibrational levels (4-3 and 3-2) of $\mathrm{CH}$ and $\mathrm{OH}$. At the present time, of the total of almost 16,000 lines in the ATMOS solar spectrum, more than 3700 remain unidentified. Since many of the unidentified lines are relatively strong, it is probable that they are also due to transitions between undetermined 


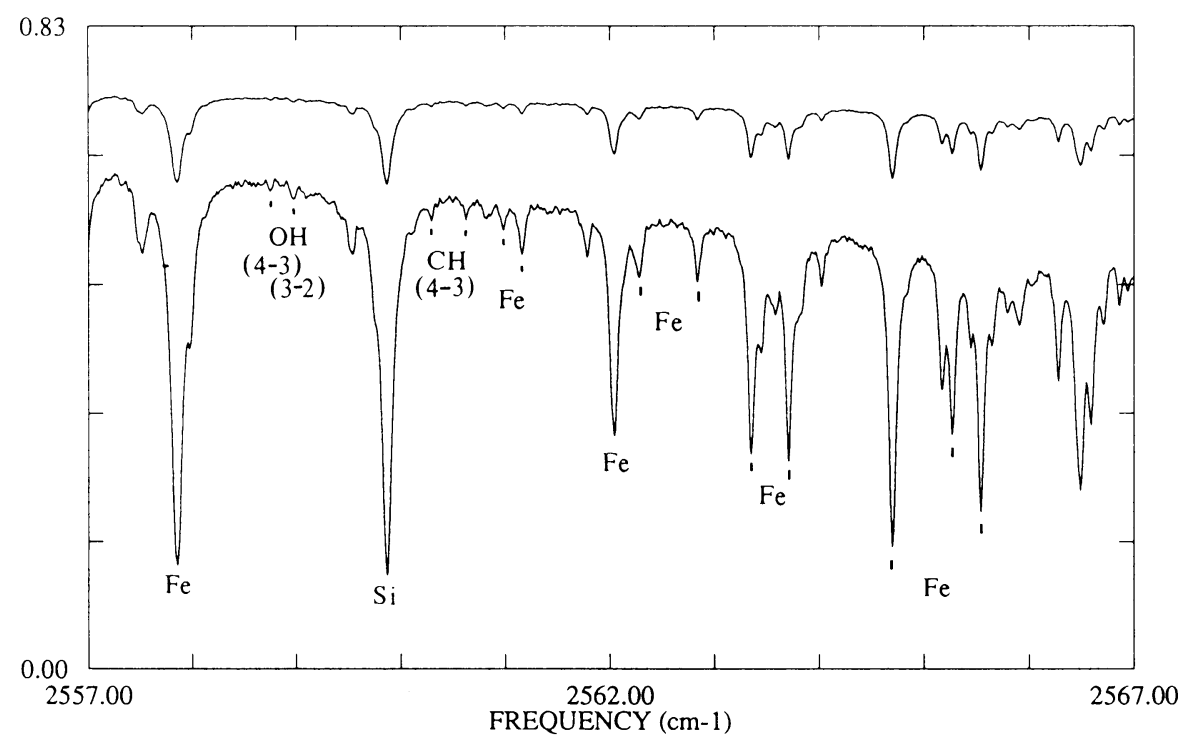

Fig. 7. In addition to the many $\mathrm{Fe}$ and $\mathrm{Si}$ lines in this region (all $4 f-5 g$ transitions), several $\mathrm{CH}$ and $\mathrm{OH}$ lines of the higher vibrational levels are found.

levels of the more abundant elements. The spectra will thus provide a valuable data base against which to validate further extensive calculations of energy levels in these species.

\section{The Future}

The solar infrared spectrum is rich in the number and variety of diagnostic features which occur in this wavelength region. This is particularly valuable because, even at high resolution, the spectrum is sufficiently uncluttered to allow detailed analysis of individual lines whose study bears on several important physical and chemical properties of the Sun. Amongst these are chemical abundances; thermal structure, radiative equilibrium and small-scale dynamics of the photosphere; and the distribution of electric and magnetic fields. However, a deeper understanding of the physics of the formation of these lines is necessary before their full potential as diagnostics of solar conditions can be realized. The ATMOS spectrum has provided the opportunity to view, for the first time, the continuous solar infrared spectrum, unbroken by the opaque regions of the Earth's atmosphere, and promises an exciting future for progress in these disciplines.

In the course of pursuing the long-term goals of the ATMOS experiment, NASA has planned to fly the instrument approximately once a year during the coming decade. ATMOS is currently included in the science payload of the first four Shut- 
tle ATLAS missions, which began in March, 1992. From the point of view of solar infrared science these flights provide an opportunity to improve and extend the spectral data obtained from the Spacelab-3 flight, and to plan solar observations that make the best use of the capabilities of the instrument to serve the interests of solar physics. Further information regarding the availability of the ATMOS data and planning for future flight opportunities can be obtained from the Principal Investigator, Dr. M. R. Gunson, at JPL. It seems clear from the results obtained so far that future mission time devoted to acquiring more data to improve the $S / N$ of the present spectra, and to making center-to-limb scans, would be of immediate value, while causing minimal perturbation to the primary atmospheric measurements. In addition, minor changes to the instrument, such as the use of smaller field apertures and a detector having a longer wavelength response (to the beamsplitter limit of $23 \mu \mathrm{m}$ ) would greatly enhance its potential value to solar science. The latter changes could be made without requiring any other modification to the interferometer or its electronic systems, and could perhaps be introduced for a single mission during which solar observations would be given priority. The cost effectiveness of this approach to obtaining data covering an unexplored wavelength region is compelling. It is hoped that for its future flights the full capabilities of the ATMOS instrument can be exploited to make observations designed expressly for these purposes.

\section{References}

Beer, R., and Norton, R. H.: 1976, J. Opt. Soc. Am. 66, 3, 259.

Brault, J. W., and Noyes, R. W.: 1983, Astrophys. J. (Letters) 263, 61.

Chang, E. S., Avrett, E. H., Mauas, P. J., Noyes, R. W., and Loeser, R.: 1991, Astrophys. J. (Letters) $379,79$.

Farmer, C. B., and Norton, R. H.: 1989, A High Resolution Atlas of the Infrared Spectrum of the Sun and the Earth Atmosphere from Space, Vol. I: The Sun, NASA Ref. Pub. 1224.

Farrenq, R., Guelachvili, G., Sauval, A. J., Grevesse, N., and Farmer, C. B.: 1991, J. Mol. Spectr. $149,375$.

Geller, M.: 1992, A High Resolution Atlas of the Infrared Spectrum of the Sun and the Earth Atmosphere from Space, Vol. III, in press.

Geller, M., Sauval, A. J., Grevesse, N., Farmer, C. B., and Norton, R. H.: 1991, Astron. Astrophys. 249, 550.

Grevesse, N., Lambert, D. L., Sauval, A. J., van Dishoeck, E. F., Farmer, C. B., and Norton, R. H.: 1990, Astron. Astrophys. $232,225$.

Grevesse, N., Lambert, D. L., Sauval, A. J., van Dishoeck, E. F., Farmer, C. B., and Norton, R. H.: 1991, Astron. Astrophys. 242, 488.

Grevesse, N., and Sauval, A. J.: 1990, in C. Jaschek and Y. Andrillat (eds.), The Infrared Spectral Region of Stars, Proc. Montpellier Colloq. (October 1990), Cambridge University Press, Cambridge.

Jefferies, J. T.: 1991, Astrophys. J. 377, 337.

Mèlen, F., Grevesse, N., Sauval, A. J., Farmer, C. B., Norton, R. H., Bredohl, H., and Dubois, I.: 1989, J. Mol. Spect. 134, 305. 